Worauf der Unterschied der Schmelztemperaturen meiner Säure and der Săure von Anwers beruht, werden die weiteren Untersachangen zeigen.

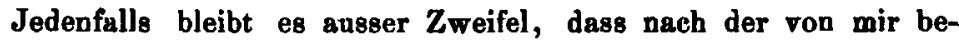
schriebenen Methode auf directem Wege keine maleĩnoïde Dimethylglutarsăure erbalten wird, während sie gemäss den Speculationen von Prof. K. A a wers erbalten werden sollte.

Nach alledem muss die überwiegende Bildung der mal. Säure nach der Methode von Prof. Zelin sky wahrscheinlich der Anziehnggkraft, welche zwei Cyangruppen auf einander ausüben, zugeschrieben werden.

Kiew, 10. December 1895.

634. E. F. Slosson: Ueber die Finwirkung von unterbromiger und unterohloriger săure ouf såureanilide.

[Vorlănfige Mittheilung a. d. Kent Chemical Laboratory, University of Chicago.]

(Eingegangen am 27. December.)

Die Acylbromamide, RCO NHBr, erfahren bekanntlich nnter dem Einfluss von wässrigen Alkalilösungen ${ }^{1}$ ) oder von alkoholischem Natriummethylat ${ }^{2}$ ) eine Umlagerung unter Bildung zuerst von Isocyanaten ${ }^{1}$ ), RN : CO, oder von Bromformamiden, BrCONHR, welche dann weiter auf etwa vorhandene Substanzen einwirken. Zum besseren Verständniss der Bedingangen, welche diese Umlagerung möglich machen, erschien es wünschenswerth, das Verhalten der für analog mit obigen Amiden gehaltenen Acylbalogenalkylamide, RCON(Hal)R, gegenüber alkalischen Lösangen und anderen umlagernden Reagentien genauer zu verfolgen, um zu bestimmen, ob unter keiner der eingehaltenen Bedingungen und auch nicht wenigstens in geringer Menge der entsprechende Austausch von $R$ gegen Halogen bewirkt werden könne. Zur Untersuchung dieser Frage, welche ich auf Veranlassung und unter der Leitung des Hrn. Dr. S tị eglitz aufgenommen habe, brachte ich zuerst Derivate der Săureanilide in Anwendung. Hier möchte ich über die Darstellung dieser Körper und vorläufig über ihr Verhalten berichten.

1) Hofmann, diese Berichte 15, 408; Hoogewerff und van Dorp, diese Berichte 20, Ref. 470.

\% Lengfeld nnd Stieglitz, Amer. Chem. Journ. 15, 215, 504. 
Acylbromaminobenzole ${ }^{1}$ ), $\mathrm{RCON} \mathrm{BrC}_{6} \mathrm{H}_{3}$, scheinen noch nie erbalten worden zu sein. Bei Reactionen, welche zu den entsprechenden Chlorverbindungen führen ${ }^{2}$ ), sowie bei solchen, welche die einfachen Acylbromamide liefern ${ }^{3}$ ), sind immer im Benzolkern substituirte Bromanilide, z. B. RCON $\mathrm{C} \mathrm{C}_{6} \mathrm{H}_{4} \mathrm{Br}$, erhalten worden. Es ist mir gelungen, die betreffenden Bromverbindangen auf einfachem Wege darzustellen. Sie gehen in der That mit der grössten Leichtigkeit in die isomeren Acylparabromanilide über.

Acetylbromaminobenzol, $\mathrm{CH}_{3} \mathrm{CONBr}_{6} \mathrm{H}_{5}$.

Fügt man bei $0^{\circ}$ eine alkalische Hypobromitlösung (aus $14-18 \mathrm{~g}$ Kali, 140-180 $\mathrm{g}$ Wasser und $16 \mathrm{~g}$ Brom) zu einer gesättigten wässrigen Lösung von Acetanilid, so bleibt die Flüssigkeit auch längere Zeit klar, es findet keine Reaction statt. Säuert man, wie bei der Darstellung von Acetylchloraminobenzols, mit verdünnter Essigsäure an, so wird reines Acetparabromanilid (Schmp. 165 ${ }^{\circ}$ ) in quantitativer Ausbeute niedergeschlagen. Wird statt Essigsäure ein langsamer, gat vertheilter Strom Kohlensibure in die kalte Mischung geleitet, so findet alsbald eine Ausscheidung von glänzenden Blättchen statt. Der Strom wird sofort abgestellt, die Krystalle schnell abfiltrirt, mit geringen Mengen kaltem Wasser gewaschen und auf Thonplatten getrocknet. Durch die Ausscheidung von Jod aus Jodkaliumlösung und durch die Entwicklang von Stickstoff aus Ammoniak wurde die Gegenwart des gesuchten Acetylbromaminobenzols in den Blättchen erkannt. Quantitative Bestimmungen des nach der Gleichung

$$
\mathrm{CH}_{3} \mathrm{CONBr} \mathrm{C}_{6} \mathrm{H}_{5}+2 \mathrm{HI}=\mathrm{CH}_{3} \mathrm{CONHC}_{6} \mathrm{H}_{5}+\mathrm{I}_{2}+\mathrm{HBr}
$$

ausgeschiedenen Jods zeigten, dass immer nur $60-70$ pCt. der Substanz Acetylbromaminobenzol war; das Uebrige wurde als Acetparabromanilid (Schmp. 165 ) in der von angesänerter Jodkaliumlösung unverändert gelassenen Substanz erkannt.

Analyse: Ber. für $\mathrm{CH}_{3} \mathrm{CONBrC}_{8} \mathrm{H}_{5}$.

$$
\begin{array}{ll}
\text { Procente: } & \mathrm{Br} 37.31 . \\
\text { Gef. } \quad \text { (actives) } & \text { 22.43, 21.13, 26.66. }
\end{array}
$$

Die Anwendung von Borsăure und eines grossen Ueberschusses an Hypobromitlösung fährte zu einer viel reineren Verbindung in sehr guter Ausbeute. Eine gesättigte Lösung von Borsăure in Wasser wird der Mischung von Acetanilid and alkalischer Hypobromitlösung hinzagefügt, bis beim anhaltenden Schütteln gerade ein Niederschlag ent-

1) Die Nomenclatar entspricht der von Beilstein in seinem Handbuch angewandten, z. B. S. 362, Bd. II. Vergl. dagegen Seliwanow, diese Berichte 25, 3618.

2) J acoby, Journ. prakt. Chem. [2] 37, 50.

T) Seliwanow, diese Berichte 26, 423. 
steht; dieser wurde wie oben angegeben weiter behandelt. Die Substanz besteht fast zu 90 pCt. aus Acetylbromaminobenzol:

Analyse: Gef. Procente (actives): Br 30.50, 32.09.

Beim Titriren des Jods mit Thiosulfat blieb eine geringe Menge Acetparabromanilid zurück; das nach obiger Gleichung gebildete Acetanilid wurde aus der entstandenen Lösung mit Aether ausgezogen und am Schmelzpunkt $\left(12^{\circ}\right)$ erkannt.

Acetylbromaminobenzol bildet sehr schwach gelbliche Blättchen, welche beim langsamen Ausscheiden mit Borsäure aus viereckigen Sternchen bestehen. Es schmilat bei $75-80^{\circ}$, erstarrt fast sofort wieder und schmilzt dann wieder bei $165^{\circ}$; es ist also beim Erhitzen in Acetparabromanilid übergegangen. Beim Kochen mit Wasser findet dieselbe Umwandlung augenblicklich statt. Der feste Körper erleidet diese Umsetzung ziemlich rasch bei gewöhnlicher Temperatur in Gegenwart der geringsten Menge Feuchtigkeit (schon in wenigen Standen ist die Umsetzung zu Ende). Entsprechend den Gleichungen:

$\mathrm{CH}_{3} \mathrm{CONBr} \mathrm{C}_{6} \mathrm{H}_{5}+\mathrm{H}_{2} \mathrm{O} \leftrightarrows \mathrm{CH}_{3} \mathrm{CONHC}_{6} \mathrm{H}_{5}+\mathrm{HOBr}{ }^{1)}$ und $\mathrm{CH}_{3} \mathrm{CONHC} \mathrm{H}_{5}+\mathrm{HOBr}=\mathrm{CH}_{3} \mathrm{CONHC}_{6} \mathrm{H}_{4} \mathrm{Br}+\mathrm{H}_{2} \mathrm{O}$ genügt eine Spur Wasser, das labile Acetylbromaminobenzol in das stabile Acetparabromanilid zu verwandeln. In Folge dieser Veränderlichkeit ist das anf nassem Weg dargestellte Acetylbromaminobenzol stets schon von etwas Acetparabromanilid begleitet. Unter Anwendung eines wasserfreien, viel umständlicheren Verfahrens hoffe ich, die Verbindung in absolut reinem Zustand zu erhalten. In der Zurückverwandlung in Acetanilid durch Jodwasserstoffsäure oder Ammoniak und in dem Uebergang in Acetparabromanilid beim Erhitzen verbält es sich analog wie Acetylchloraminobenzol und es ist daher kein Zweifel möglich über die Natar der Verbindung. In Bezng auf Beständigkeit steht es 2 wischen den bekannten Chlor- und Jodverbindungen dieser Art: Acetylchoraminobenzol ist beständig sogar gegen kochendes Wasser ${ }^{2}$ ), während bei der Darstellung ron Formyljodaminobenzol Wasser sorgfältig ausgeschlossen sein muss '). Acetylbromaminobenzol lässt sich in Gegenwart von Wasser erhalten, geht aber langsan schon bei gewöhnlicher Temperatur durch Wasser in Acetparabromanilid über. Auch beim Erhitzen sind die Chlorderivate beständiger: Acetylchloraminobenzol schmilzt bei $91^{\circ}$, giebt aber erst bei $172^{\circ} p$-Acetchloranilid ${ }^{2}$ ); auch Formylehloraminobenzol (s. unten) kann obne Zersetzung weit über $100^{\circ}$ erhitzt werden. Die entsprechenden Bromderivate werden schon bei $60-80^{\circ}$ in die Acyl$p$-bromanilide umgewandelt.

1) Seliwanow, loc. cit.

9) Bender, diese Berichte 19, 2272.

3) Comgtock und Kleeberg, Americ. Chem. Jouro. 12, 500. 
Formylbromaminobenzol, $\mathrm{HCONBr} \mathrm{C}_{6} \mathrm{H}_{6}$,

wird ans Formanilid, Kaliumhypobromid und Borsăure erhalten, gemengt mit etwa 20 pCt. $p$-Bromformanilid.

Analyse: Ber. für $\mathrm{HCONBrC}_{6} \mathrm{H}_{5}$.

Procente: $\quad$ Br 39.94.

Gef. \ (activ.) » 31.35 .

Die Verbindung bildet sehr blass gefärbte Krystalle, schmilzt bei $55-57^{\circ}$, erstarrt und schmilat wieder bei $11^{\circ}$, unter Umwandlung in Form-p-bromanilid (Schmp. 1190). Sie ist löslich in Aether, Chloroform und Alkohol, wenig löslich in Ligroïn. In Berührung mit Wasser findet schnell Zersetzung statt.

Ausser der Bildung der genannten Bromverbindungen wurde auch die Darstellung der beständigeren Acylchloraminobenzole für den eingangs erwähnten $Z_{\text {weck }}$ untersucht.

\section{Acetylchloraminobenzol, $\mathrm{CH}_{3} \mathrm{CON} \mathrm{Cl} \mathrm{C}_{6} \mathrm{H}_{5}$,}

ist bereits von $B$ ender ${ }^{1}$ ) erbalten worden durch Versetzen einer wässrigen Lösung von Acetanilid mit Chlorkalklösung und Ansäuern mit Essigsäure. Bei der Darstelluug dieses und analoger Körper in grösseren Mengen erhielt ich die besten Resultate durch Umgehen des Säurezusatzes unter Anwendung einer wässrigen Lösung der unterchlorigen Säure. In eine eiskalt gehaltene 10-procentige Lösung von Natriumcarbonat wurde Chlor bis zur Süttigung geleitet und der Ueberseluuss an gelöstem Chlor durch einen Laftstrom entfernt. Beim Versetzen einer gesättigten wässrigen Lösung von Acetanilid mit einem Ueberschuss dieser Hypochloridlösung wurde eine quantitative Ausbeute rein weissen Acetylcbloraminobenzols vom Schmp. $91^{\circ}$ erbalten.

Eine Chlorbestimmung wurde mittels angesäuerter Jodkaliumlösung ausgeführt.

Analyse : Ber. für $\mathrm{CH}_{3} \mathrm{CONClC}_{6} \mathrm{H}_{5}$.

$$
\text { Procente: } \mathrm{Cl} 20.91 .
$$

Gef. " $\quad 20.83$.

Durch Alkalien unter verschiedenen Bedingungen findet kein Austausch ron Methyl gegen Chlor statt, sondern es tritt Verseifung zu Acetanilid und Alkalibypochlorit fast quantitativ ein.

Formylchloraminobenzol, $\mathrm{HCON} \mathrm{ClC}_{6} \mathrm{H}_{5}$,

auf eben demselben Wege dargestellt, bildet weisse Krystallblättchen, welche bei $43-44^{\circ}$ schmelzen. Es zeigt eine für diese Körperklasse bemerkenswerthe Beständigkeit beim Erhitzen; man kann es über $100^{\circ}$ erhitzen, ohne dass Zersetzung oder Umwandlung in Form-

2) Bender, loc. oit. 
p-chloranilid stattfindet; beim Abkühlen wird es durch ein Kryställchen zum Krystallisiren gebracht und schmilzt von Nenem bei 43 bis 440. Bei höheren Temperaturen wird der Körper zersetzt.

Analyse: Ber. für $\mathrm{HCONClC}_{6} \mathrm{H}_{5}$.

Procente: $\mathrm{Cl} 22.76$.

Gef. > " $22.39,22.40$.

Man kann Formylchloraminobenzol aus Aether oder verdünnter Essigsäure umkrystallisiren; A mmoniak, Jod wasserstoffsäure oder heisser absoluter Alkohol reduciren es zu Formanilid; Salzsäure verwandelt es in der Wärme in das noch unbekannte

\section{Formparachloranilid, $\mathrm{HCON} \mathrm{CC}_{6} \mathrm{H}_{4} \mathrm{Cl}$.}

Zum Vergleich wurde dasselbe auch aus Ameisensäure und $p$-Chloranilin dargestellt. Auf beiden Wegen wird diese Verbindung in der Form von weissen Krystallen erbalten mit dem Schmelzpunkt $101^{\circ}$. Eine Mischung beider Substanzen zeigte denselben Schmelzpunkt, sie sind also identisch.

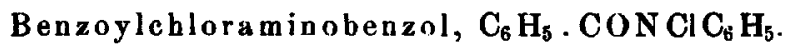

Die Unlöslichkeit von Benzanilid in Wasser bereitete der Darstellung des Chlorderivates einige Schwierigkeiten. Die Behandlung einer Essiglösung von Benzanilid mit einer wässrigen Lösung von Chlorkalk gab unbefriedigende Resultate. Der Körper wird erbalten, wenn man eine gesättigte Lösung ron Benzanilid in Alkohol tropfenweise zu einem grossen Ueberschuss einer auf dem oben beschrjebenen Wege uus Natriumcarbonat und Chlor bereiteten Lösung von unterchloriger Sãare hinzufügt. Es fällt sofort Benzoylchloraminobenzol aus, mit einer nur geringen Menge Benzanilid. Es ist löslich in Alkohol, Aether und Ligroïn und wird aus der letzten Lösung in Nadeln vom Schmp. $78-80^{\circ}$ erbalten.

Analyse: Ber. für $\mathrm{C}_{8} \mathrm{H}_{5} \mathrm{CONClC}_{6} \mathrm{H}_{5}$.

$$
\begin{array}{cc}
\text { Procente: } & \mathrm{Cl} 15.29 . \\
\text { Gef. } & \text { (act.) } \$ 14.27 .
\end{array}
$$

Anmerkung. Bei allen bisher untersuchten Verbindungen von der Form RCONHHal' (Hal' $=\mathrm{Br}$ oder $\mathrm{Cl}$ ) findet ein der sogen. ,Beckmann'schen Umlagerung، analoger ${ }^{1}$ ) Austausch von $\mathbf{R}$ gegen Hal' äusserst leicht statt durch die Einwirkung von wässriger ${ }^{2}$ ) oder alkoholisch er ${ }^{3}$ ) Alkalilösung. In einem von einem meiner Schüler, Hrn. Mc Coy, an Salicylamid beobachteten Falle geht diese Um-

1) Hoogewerff und Van Dorp, Rec. trav. chim. 8, 173; 9, 33 etc.

$\eta$ Hofman, diese Berichte 15, 410 u. s. w.; Hoogewerff und $\mathbf{\text { an }}$ Dor p, loc. eit.

3) Lengfeld and Stieglitz, Americ. Chem. Journ. 15, 215, 504.

Berichte d. D. chem. Gesellschaft. Jahre- XX VIII. 
lagerung augenblicklich schon bei $0^{0}$ oder $-10^{0}$ vor sich. Dagegen ist bis jetzt weder ron Hrn. Slosson, noch von anderen Cbemikern ${ }^{1}$ ), welche mit derartigen Verbindungen gearbeitet haben, bei den für analog mit obigen Körpern gehaltenen Verbindungen, RCON(Hal) $R^{\prime}$, dieser Austausch von $\mathbf{R}$ gegen Halogen jemals beobuchtet worden. Dieser Unterschied im Verbalten scheinbar so nahe stehender Körper, wie RCON(Hal) H und RCON(Hal)R', ist so auffallend, dass es geboten erscheint, nuch dem Grunde dafür zu suchen: entweder sind die Verbindungen wirklich nicht analnger Art oder die >Beckmann'sche Umlagerung « ist von gewissen Bedingungen abhängig, welche in diesen zwei Körperklassen zum Vorschein kommen. Während wohl die meisten Chemiker ${ }^{2}$ ) die Säurehalogenalkylamide als $\mathrm{RCON}(\mathrm{Hal}) \mathrm{R}$ auffassen, ist ron Comstock ${ }^{3}$ ) neuerdings auch die isomere Form $\mathrm{RC}(\mathrm{NR})(\mathrm{OHal})$ in Betracht gezogen worden, bei welcher die Umlagerung ausgeschlossen wäre. Gegen diese Auffassung ist aber zu bemerken, dass gerade bei den Reactionen, auf welche Comstock seine Ansicht gründet, nämlicb bei der Einwirkung von Alkyljodiden ${ }^{4}$ ) bezw. von Halogenen ${ }^{\text {s) }}$ auf die Alkali- und Silbersalze Bernsteinsäureimid sich den Säureaniliden in jeder Beziehung gleich verhält und ein Chlor- und Bromimid bildet, welche jedenfalls die Constitution $\left(\mathrm{CH}_{2} \mathrm{CO}\right)_{2}$ (NHal) besitzen: sie erfahren nämlich durch wässriges Alkali ${ }^{6}$ ) und durch Natriummethylat ${ }^{7}$ ) nach Verseifung zu den entsprechenden Succinaminsäurederivaten die Beckmann'sche Umlagerung in vollkommen glatter Weise. Phtalimid ${ }^{6}$ ) verhält sich ebenso. Erweisen sich aber die Säurehalogenalkylamide and die Säurehalogenamide wirklich als andloge Verbindungen - und dies ist recht wahrscheinlich - dann muss die ,Beckmann'sche Umlagerunge von gewissen, hier zum Vorschein kommenden Bedingungen abbängen, auf deren Betrachtung hier nicht eingegangen werden soll, da die experimentellen Cintersuchungen darüber erst begonnen sind.

\section{J. Stieglitz.}

1) Bender, diese Berichte 19, 2272; Tscherniak und Norton, Bull. soc. chim. 30, 106; Einhorn und Lauch, Ann. d. Chem. 243, 343.

') Bender, loc. cit.; Tscherniak und Norton, loc. cit.; Soliwanow, diese Berichte 26, 989; Meyer und Jacobsen's Lehrbuch II, 183, u. s. w.

3) Comstock, Americ. Chem. Journ. 12, 500.

4) Comstock und Wheeler, Americ. Chem. Journ. 13, 521. loc. eit.

5) Bunge, Ann. d. Chem., Suppl. 7, 119; Lengfeld und Stieglitz

5) Hoogewerff und van Dorp, diese Berichte 24, Ref. 966.

i) Lengfeld und Stieglitz, loc. cit. 